\title{
Streptomyces vietnamensis GIMV4.0001: a granaticin-producing strain that can be readily genetically manipulated
}

\author{
Ming-Rong Deng ${ }^{1,2}$, Jun Guo ${ }^{2}$ and Hong-Hui $\mathrm{Zhu}^{2}$ \\ The Journal of Antibiotics (2011) 64, 345-347; doi:10.1038/ja.2011.3; published online 9 February 2011
}

Keywords: conjugation; genetic manipulation; granaticin; PCR-targeted disruption; Streptomyces vietnamensis

Granaticin belongs to a class of aromatic polyketides, benzoisochromanequinone antibiotics, among which actinorhodin is the most well-known member. The biosynthetic gene cluster of actinorhodin have been intensively studied and serves as a model for studying the type II polyketide synthatase (PKS) pathways. Granaticin has a similar basic skeleton but unique fine structure compared to actinorhodin (Figure 1). The opposite stereochemistry of the pyran ring and the unusual sugar attachment presented in granaticin structure has drawn much attention among chemical and biochemical researchers. The granaticin biosynthetic gene cluster (gra) was identified originally from Streptomyces violaceoruber Tü22, ${ }^{1}$ and the functions of most biosynthetic genes were assigned based on sequence homology. After that, only a few functional studies have been carried out on this cluster. $^{2-5}$ The functions of several gra genes remain totally unknown. Knowledge about how the production is regulated is still limited. The fact that $S$. violaceoruber Tü22 is recalcitrant to plasmid transformation $^{1}$ has imposed a significant barrier to its study.

S. vietnamensis GIMV4.0001 (CCTCC M 205143, hereafter referred as GIMV4.0001), the type strain of a newly designated streptomycete species by our laboratory, ${ }^{6}$ was found to be a novel granaticin producer, and the gra cluster was sequenced by a sequential cloning strategy (unpublished result, GenBank accession number: GU233672). In this study, we report the establishment of an efficient and stable conjugation system between Escherichia coli and GIMV4.0001 and applying a modified PCR-targeted disruption method to GIMV4.0001.

All strains and plasmids used in this study are listed in Supplementary Table S1. To screen putative exconjugants derived from GIMV4.0001, we first carried out an antibiotic sensitivity test using an agar diffusion method (Supplementary Information, Materials and methods). All the plates containing GIMV4.0001 showed clear zones of inhibition around the holes with apramycin, kanamycin, thiostrepton, streptomycin or spectinomycin at $10 \mu \mathrm{g} \mathrm{ml}^{-1}$, suggesting that there were adequate choices of selectable marker for genetic manipulation of GIMV4.0001.

Two kinds of plasmids, auto-replicating pHZ1358 and integrative pSET152, were used to test the feasibility to introduce foreign DNA into GIMV4.0001. The basic procedures of conjugation between E. coli and GIMV4.0001 were as described by Kieser et al., ${ }^{7}$ but some modifications were made (Supplementary Information, Materials and methods). The optimal medium for conjugation was screened. Exconjugants were confirmed by PCR amplification with an aac3(IV)-specific primer pair (for pSET152; Supplementary Information, Table S2) or by plasmid isolation and restriction mapping (for pHZ1358). For pSET152, putative exconjugants were obtained from YD, SFM, YMS and Gauze's synthetic agar, but the efficiency differed. The conjugation frequency for YD, SFM, YMS and Gauze's synthetic agar were $8 \times 10^{-4}, 3 \times 10^{-4}, 5 \times 10^{-6}$ and $2 \times 10^{-5}$, respectively. For pHZ1358, putative exconjugants were obtained only from YD medium, and the frequency was $2 \times 10^{-8}$. These results suggested that YD was an optimal medium for conjugation. Pre-germination time of spores and incubation time before overlaying antibiotics were subsequently subject to optimization. Pre-germination time had a limited influence on the transfer efficiency, whereas incubation time before overlaying antibiotics was critical for conjugation. Incubation of about $12 \mathrm{~h}$, at the stage when sporulation just started, was the best. When the incubation time was shortened to $10 \mathrm{~h}$, no exconjugants were observed for $\mathrm{pHZ1358.} \mathrm{Prolonged} \mathrm{incubation} \mathrm{also} \mathrm{could} \mathrm{cause} \mathrm{failure} \mathrm{due} \mathrm{to} \mathrm{the}$ hydrophobic nature of Streptomyces spores. The conjugation experiment with optimized conditions was conducted repeatedly. The results demonstrated that an efficient and stable conjugation system between E. coli and GIMV4.0001 had been established.

A modified PCR-targeted disruption method $^{8}$ was further applied to GIMV4.0001 to explore the possibility of performing functional study in vivo with this strain. A 7-kb PCR product containing the minimal PKS genes (orf1, 2, 3) for granaticin and flanking sequences,

${ }^{1}$ School of Biological Science and Engineering, South China University of Technology, Guangzhou, China and ${ }^{2}$ Guangdong Provincial Key Laboratory of Microbial Culture Collection and Application, Guangdong Open Laboratory of Applied Microbiology, Guangdong Institute of Microbiology, Guangzhou, China Correspondence: Dr H-H Zhu, Guangdong Institute of Microbiology, No. 100 Xianliezhong Road, Guangzhou 510070, China. E-mail: zhuhonghui66@yahoo.com.cn

Received 1 October 2010; revised 23 December 2011; accepted 3 January 2011; published online 9 February 2011 
amplified from the genomic DNA of GIMV4.0001 with primers XgraF and XgraR (Supplementary Information, Table S2), was cloned into the pCR2.1 vector. The resulting plasmid (pCR-Xgra-minipks) was substituted for the genomic library cosmid and then was introduced into E. coli BW25113/pIJ790, serving as the target plasmid for PCRtargeted disruption. The extended aac(3)-IV/oriT cassette amplified from pIJ773 with primers TAR1F and TAR1R (Supplementary Information, Table S2) was electro-transformed into E. coli BW25113/ pIJ790/pCR-Xgra-minipks. The minimal PKS genes (orf1, 2, 3) for granaticin on pCR-Xgra-minipks would be replaced by the aac(3)-IV/ oriT cassette after induction of the $\lambda$ red genes. The mutant target plasmid (pCR-Xgra-minipks-Apra) was confirmed by restriction ana-<smiles>CC1OC(CC(=O)O)CC2=C1C(=O)c1c(O)c(-c3cc(O)c4c(c3O)C(=O)C3=C(CC(CC(=O)O)OC3C)C4=O)cc(O)c1C2=O</smiles>

Figure 1 Structures of granaticin and actinorhodin.

a

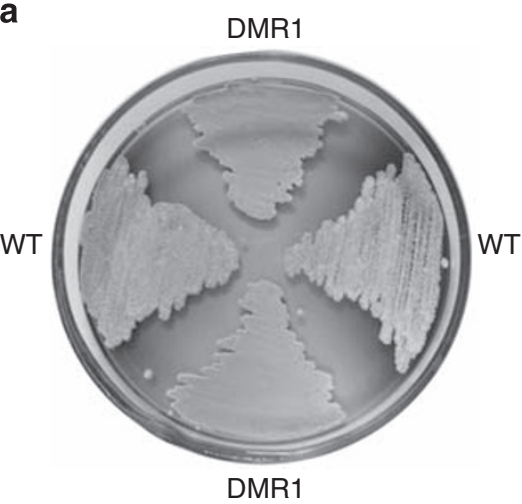

C

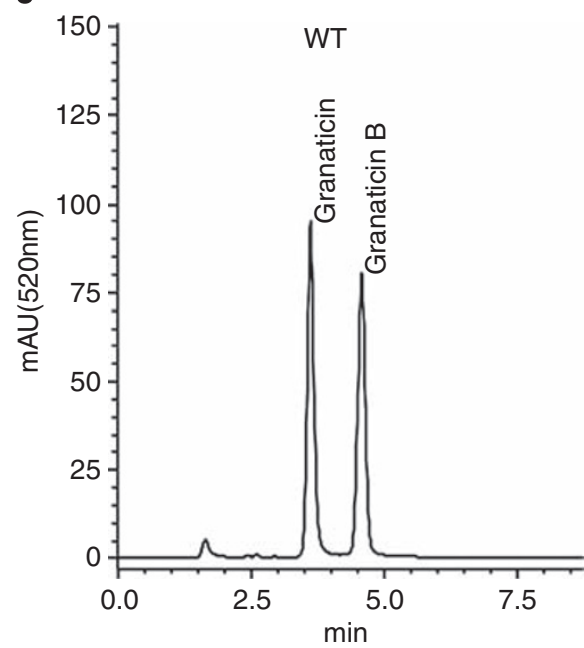

lysis and then introduced into E. coli ET12567/pUZ8002. Intergeneric conjugation was performed on YD agar medium. The promoter upstream of the neo gene on pCR2.1 was still active in GIMV4.0001. This allowed the use of kanamycin to discriminate between double crossover mutant and single crossover mutant. Double crossover exconjugants were kanamycin sensitive and apramycin resistant. The screening and purification of the mutant were carried out and allowed the isolation of the double crossover non-granaticin-producing mutant DMR1. DMR1 showed growth and morphological characteristics identical to those of the wild-type strain, while the ability to produce granaticin was completely abolished (Figure 2a). The genomic DNA of DMR1 was subject to a PCR confirmation procedure. Amplicons of $708 \mathrm{bp}$ (ApraF/ApraR), $3387 \mathrm{bp}$ (XgraF/ApraR), 2911 bp (ApraF/XgraR) and 5590 bp (XgraF/XgraR) from the mutant were detected. In contrast, only a fragment of $7012 \mathrm{bp}$ (XgraF/XgraR) from the wild strain was observed (Figure $2 b$ ). This agreed well with the predicted replacement of the minimal PKS genes with the aac(3)-IV cassette. The inability of DMR1 to produce granaticin and granaticin $\mathrm{B}$ was further confirmed by HPLC analysis. No peak corresponding to granaticin and granaticin B was observed in the DMR1 (Figure 2c). These results suggested that

b
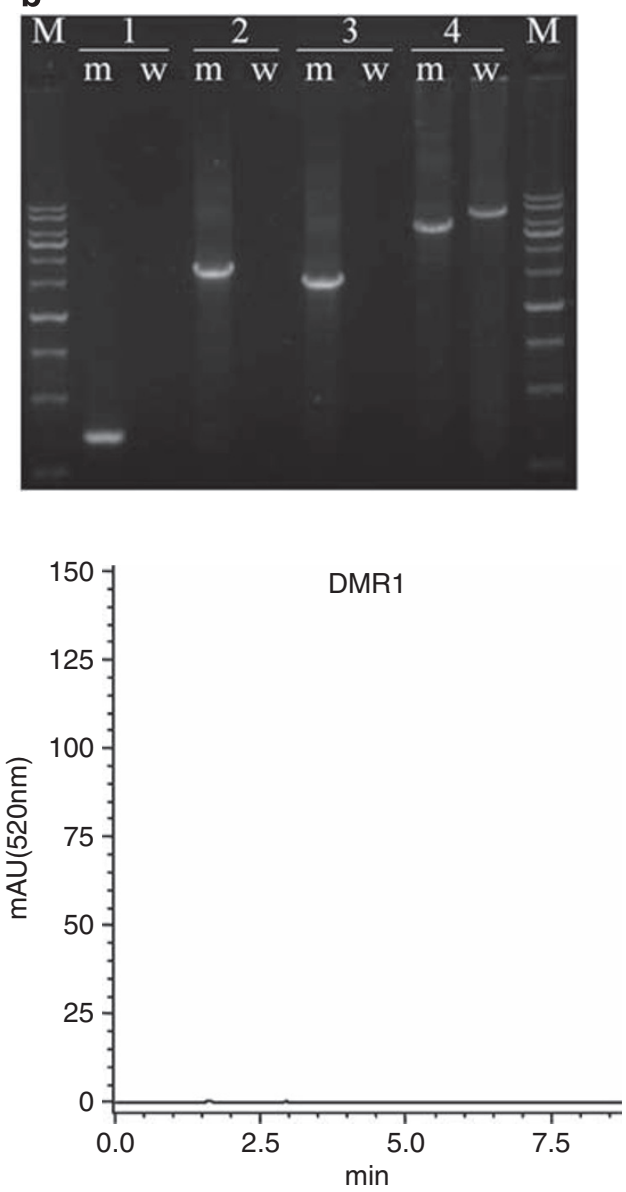

Figure 2 Construction of the granaticin-deficient mutant. a, S. vietnamensis GIMV4.0001 wild-type (WT) and granaticin-deficient mutant DMR1 grown on Gauze's synthetic agar. b, PCR verification of the gene type of the mutant DMR1. M, $1 \mathrm{~kb}$ ladder marker; $1-4$, PCR products with primer pairs of ApraF/ApraR, XgraF/ApraR, ApraF/XgraR and XgraF/XgraR, respectively. ' $m$, w' below the white lines indicates that the templates were the genomic DNA of the DMR1 mutant and wild-type strains, respectively. c, HPLC analysis of ethyl acetate extracts from S. vietnamensis GIMV4.0001 WT and DMR1 mutant strains. HPLC is described in the Supplementary Information 'Materials and methods' section. The retention times of granaticin and granaticin B are 3.621 and $4.574 \mathrm{~min}$, respectively. 
the disruption of the minimal PKS genes for granaticin by the modified PCR-targeted disruption method had been successfully made in GIMV4.0001. The results also validated the feasibility of substitution of plasmids derived from the TA cloning vector pCR2.1 for the genomic library cosmid when the genomic library cosmids were unavailable.

In conclusion, we have established an efficient and stable conjugation system between E. coli and GIMV4.0001. On the basis of this conjugation system, a granaticin-deficient mutant of GIMV4.0001 was successfully made by application of a modified PCR-targeted disruption method. To our knowledge, this is the first report for the efficient genetic manipulation on the granaticin-producing strain, which paves the way to studying the mechanisms of regulations and biosynthesis of granaticin in vivo.

\section{ACKNOWLEDGEMENTS}

This work was supported by a grant from the International Science and Technology Cooperation Programs of China (2008DFA31560), the Chinese Academy of Sciences for Key Topics in Innovation Engineering (KSCX2-YW-G075-11), the Science and Technology Innovation Programs of Guangdong Academy of Sciences (cx200702) and the Foundation for Young Scientists of Guangdong Academy of Sciences (qnij20092). We are grateful to Heather McCartney at John Innes Centre for providing plasmids pIJ773 and pIJ790, Professor Zi-Xin Deng and De-Lin You at Shanghai Jiaotong University for providing strains ET12567/pUZ8002 and BW25113, plasmids pHZ1358 and pSET152, and helpful advice.

1 Ichinose, K., Bedford, D. J., Bibb, M. J., Revill, W. P. \& Hopwood, D. A. The granaticin biosynthetic gene cluster of Streptomyces violaceoruber Tü22: sequence analysis and expression in a heterologous host. Chem. Biol. 5, 647-659 (1998).

2 Tornus, D. \& Floss, H. G. Identification of four genes from the granaticin biosynthetic gene cluster of Streptomyces violaceoruber Tü22 involved in the biosynthesis of L-rhodinose. J. Antibiot. 54, 91-101 (2001).

3 Taguchi, T., Ebizuka, Y., Hopwood, D. A. \& Ichinose, K. A new mode of stereochemical control revealed by analysis of the biosynthesis of dihydrogranaticin in Streptomyces violaceoruber Tü22. J. Am. Chem. Soc. 123, 11376-11380 (2001).

4 Ichinose, K., Taguchi, T., Bedford, D. J., Ebizuka, Y. \& Hopwood, D. A. Functional complementation of pyran ring formation in actinorhodin biosynthesis in Streptomyces coelicolor A3(2) by ketoreductase genes for granaticin biosynthesis. J. Bacteriol. 183, 3247-3250 (2001).

5 Draeger, G., Park, S. H. \& Floss, H. G. Mechanism of the 2-deoxygenation step in the biosynthesis of the deoxyhexose moieties of the antibiotics granaticin and oleandomycin. J. Am. Chem. Soc. 121, 2611-2612 (1999).

$6 \mathrm{Zhu}, \mathrm{H}$. H. et al. Streptomyces vietnamensis sp. nov., a streptomycete with violet blue diffusible pigment isolated from soil in Vietnam. Int. J. Syst. Evol. Microbiol. 57, 1770-1774 (2007).

7 Kieser, T., Bibb, M. J., Buttner, M. J., Chater, K. F. \& Hopwood, D. A. Practical Streptomyces Genetics (John Innes Foundation, Norwich, 2000).

8 Gust, B., Challis, G. L., Fowler, K., Kieser, T. \& Chater, K. F. PCR-targeted Streptomyces gene replacement identifies a protein domain needed for biosynthesis of the sesquiterpene soil odor geosmin. Proc. Natl Acad. Sci. USA 100, 1541-1546 (2003)

Supplementary Information accompanies the paper on The Journal of Antibiotics website (http://www.nature.com/ja) 